\title{
Naturheilkunde in Leitlinien: David Sackett und die frischen Frisuren
}

\author{
Detmar Jobst
}

Stellvertretender Direktor und Sprecher der AG Komplementärmedizin in der DEGAM, Mitglied der Leitlinienkonferenz der DEGAM, Institut für Hausarztmedizin, Universitätskliniken Bonn, Bonn, Deutschland

Die Sackett'sche Idee der evidenzbasierten Medizin (EBM) hat zu forschungsbasierten Therapieentscheidungen geführt, Lehrmeinungen und Postulate infrage gestellt und den Wissenstransfer beschleunigt. Ein neues Zeitalter der Aufklärung in der Medizin wurde eingeläutet. Man könnte sagen: Alte Zöpfe wurden und werden im Licht aktueller wissenschaftlicher Erkenntnisse abgeschnitten, die Meinung von Opinion Leaders und Experten muss sich hinten anstellen. Auch Naturheilverfahren (NHV) wurden davon erfasst.

Dann bewegte sich die EBM in eine problematische Richtung: Ihre Auslegung ging zunehmend mit starrem Blick auf Meta-Analysen und Reviews einher. Beschrieben hat David Sackett die Anwendung der EBM jedoch so: «...in making decisions about the care of individual patients, the practice of evidence based medicine means integrating individual clinical expertise with the best available external clinical evidence from systematic research» [1]. Die Ergebnisse von Meta-Analysen und Reviews bestimmen heute die Kernaussagen von Leitlinien, die wiederum in Lehrbüchern und vor Gerichten zu Richtlinien avancieren. Dabei sollten Leitlinien Wissenskorridore bilden, erfüllt von den besten wissenschaftlichen Erkenntnissen, von der Expertise der ärztlichen Anwender und den Bedürfnissen der Patienten. Die Sackett'sche EBM sieht diese Trias vor! Und NHV sowie die Komplementär- und Alternativmedizin (CAM) in Leitlinien funktionieren aus meiner Sicht genau so am allerbesten: als evidenzgestützte Wissenskorridore, angewendet von geschulten und kundigen Ärzten, in gutem Austausch mit den Patienten und zu deren Wohl.

Im Betrieb der Arbeitsgemeinschaft der Wissenschaftlichen Medizinischen Fachgesellschaften e.V., der Dachorganisation für das Regelwerk der Leitlinienerstellung, gilt naturgemäß das geschriebene Wort - der Austausch mit den Patienten und ihre Behandlung finden in Kliniken und Praxen statt. Die ärztlichen Autoren und Vertreter von NHV und CAM können sich trotzdem glücklich schätzen, am Leitlinienschaffen teilzuhaben: Durch die geforderten Evidenzbeweise geben sie Impulse an das Forschungsgeschehen, frischen damit und durch umfängliche Literaturrecherchen die Kenntnisse über die tradierten Lehren auf und stellen NHV und CAM im besten Fall gleichberechtigt in einer Leitlinie dar, direkt neben den Methoden der Mainstream-Medizin. Jedenfalls hat der «body of evidence», der weltweite Wissensschatz von NHV und CAM, in erfreulichem Umfang zugenommen.

Im ungünstigen Fall müssen die Vertreter von NHV und CAM gegen Vorurteile von Mitautoren kämpfen. Zwar mag formal eine Untersuchung über Blutegel dem hohen Evidenzlevel 1b (z.B. für die Linderung von Kniearthrose-Schmerzen) entsprechen. Auch mag der Blutegel als Medizinprodukt apothekengängig und verordnungsfähig, sogar abrechenbar nach der Gebührenordnung für Ärzte sein. Doch warum erhält er in der Leitlinie nur die schwächste Empfehlungsstärke C?

\section{KARGER}

Fax +497614520714
Prof. Dr. Detmar Jobst

Institut für Hausarztmedizin, Universitätskliniken Bonn

Sigmund-Freud-Straße 25, 53127 Bonn, Deutschland

detmar.jobst@ukb.uni-bonn.de 
Die Voreingenommenheit gegen NHV und CAM hat viele Ursachen. Eine der wichtigsten sind die Curricula im Medizinstudium, die von einer tiefgehenden technischen Spezialisierung geprägt werden. Wenig Raum ist dort vorgesehen für patientenorientierte Medizin, wie sie mir recht typisch für NHV und CAM zu sein scheint.

Also stellen sich mehrere Aufgaben, denen sich Forscher, Hochschullehrer, Ärzte und Leitlinienautoren widmen müssen: das Schaffen von Evidenz durch Forschungsergebnisse, Enthusiasmus in der Lehre von NHV und CAM, Expertise wie auch überzeugende Leistungen in der Patientenversorgung und Durchsetzungskraft in der Leitlinienarbeit. Die Essener Initiatoren und Ausrichter des Updates/Symposiums über Leitlinienarbeit in der komplementären Medizin haben 2015 in diesem Sinne Ergebnisse beigesteuert, die nicht verloren gehen, da sie in dieser Ausgabe der Forschenden Komplementärmedizin dokumentiert werden. Damit ist ein weiterer Schritt getan, um die Naturheilkunde auf neuzeitliche Füße zu stellen und aus altmodischen Zöpfen praktische und frische Frisuren zu machen - ehe andere sie abschneiden. Ärztliche Expertise in der komplementären Medizin wird jedoch trotz und wegen der Leitlinienarbeit nicht in den Hintergrund treten, sondern im Gegenteil zum Patientenwohl angewendet werden - ganz im Sackett'schen Sinne. 
Im Gasteditorial von Detmar Jobst

Forsch Komplementmed 2016;23:147-148 (DOI: 10.1159/000447440)

\section{«Naturheilkunde in Leitlinien: David Sackett und die frischen}

Frisuren "

fehlte leider die Literaturangabe. Sie lautet:

1 Sackett DL, Rosenberg WM, Gray JA, Haynes RB, Richardson WS: Evidence based medicine: what it is and what it isn't. BMJ 1996;312:71-72. 